IPMU-12-0066

KEK-TH-1538

August 3, 2018

\title{
Search for the Top Partner at the LHC using Multi-b-Jet Channels
}

\author{
Keisuke Harigaya $^{(a, b)}$, Shigeki Matsumoto ${ }^{(a)}$, \\ Mihoko M. Nojiri ${ }^{(c, d, a)}$ and Kohsaku Tobioka ${ }^{(a, b)}$ \\ ${ }^{(a)}$ Kavli Institute of the Physics and Mathematics of the Universe (Kavli IPMU), \\ TODIAS, University of Tokyo, Kashiwa, 277-8583, Japan \\ ${ }^{(b)}$ Department of Physics, University of Tokyo, Bunkyo-ku 113-0033, Japan \\ (c) KEK Theory Center, Tsukuba, Ibaraki 305-0801, Japan \\ (d) The Graduate University for Advanced Studies (Sokendai), Department of \\ Particle and Nuclear Physics, Tsukuba, Ibaraki 305-0801, Japan
}

\begin{abstract}
Vector-like quarks are introduced in various new physics models beyond the standard model ( $\mathrm{SM})$ at the $\mathrm{TeV}$ scale. We especially consider the case that the quark is singlet (triplet) under the $\mathrm{SU}(2)_{L}\left(\mathrm{SU}(3)_{c}\right)$ gauge group and couples only to the third generation quarks of the SM. The vector-like quark of this kind is often called a top partner. The top partoner $t_{p}$ decays into $b W, t Z$ and $t h$. In the ATLAS and CMS collaborations, the top partner has been searched in the final states of $b W$ and $t Z$, while the search based on the decay mode $t_{p} \rightarrow$ th has not been started yet. However, the decay into th is important since it is significantly enhanced if some strong dynamics exists in the $\mathrm{TeV}$ scale. In the presence of a light higgs boson, the decay mode $t_{p} \rightarrow t h$ followed by $h \rightarrow b \bar{b}$ produces three bottom quarks. We study the sensitivity for the top partner using multi-b-jet events at the $8 \mathrm{TeV}$ run of the LHC experiment. The multi-b-jet eventss turn out to play a complementary role to the existing $t_{p} \rightarrow b W$ and $t Z$ searches by the ATLAS and CMS collaborations.
\end{abstract}




\section{Introduction}

Many new physics models at the $\mathrm{TeV}$ scale have been proposed so far to solve the hierarchy problem of the standard model (SM). Vector-like quarks, which are singlet (triplet) under the $\mathrm{SU}(2)_{L}\left(\mathrm{SU}(3)_{c}\right)$ gauge group, are often introduced in those models. One of famous examples is the little higgs model [1] which has been proposed to solve the little hierarchy problem [2]. In this model, the higgs boson is regarded as a pseudo-NB boson associated with a spontaneous symmetry breaking at $10 \mathrm{TeV}$. Explicit breaking terms are arranged to cancel quadratically divergent corrections to the higgs mass term at the 1-loop level, which stabilizes the higgs mass at $\mathcal{O}(100) \mathrm{GeV}$. This arrangement is called the collective symmetry breaking, and it requires new vector-like quarks. The mass of the vector-like quark is predicted to be less than 600 (900) GeV to realize the fine-tuning less than 10\% (5\%) level [3], which is within the reach of the Large Hadron Collider (LHC) experiment.

Another example is the extension of the minimum supersymmetric standard model (MSSM) by introducing vector-like matters [4. This extension recently attracts attention because of the latest results of the LHC experiment, where the higgs mass is suggested to be $124-126 \mathrm{GeV}$ by both the ATLAS [5] and CMS [6] collaborations. In the framework of the MSSM, it is difficult to achieve such high mass higgs boson for $M_{\mathrm{SUSY}} \sim 1 \mathrm{TeV}$ [7], leading to a little hierarchy problem. If there are new vector-like matters in the model, the higgs mass can be as heavy as $125 \mathrm{GeV}$ while keeping $M_{\text {SUSY }} \sim 1 \mathrm{TeV}$. Introduction of vector-like matters therefor allows us to set $M_{\text {SUSY }}$ as light as current experimental limits which are, for example, consistent with results of the anomalous magnetic dipole-moment of the muon [8].

In this article, we focus on the up-type vector-like quark whose hypercharge is $2 / 3$. We also postulate that the vector-like quark is interacting only with the third generation quarks of the SM in order to satisfy severe constraints from flavor changing processes [9]. The vector-like quark of this kind is often called a top partner, and the character $t_{p}$ is used to denote it in following discussions. In general, the top partner can decay into $b W, t Z, t h, t g$ and $t \gamma$. The decay modes $t_{p} \rightarrow b W, t Z$ and th are dominant because the other modes $t_{p} \rightarrow t g$ and $t_{p} \rightarrow t \gamma$ only appears in the 1-loop diagrams due to the gauge invariance. The top partner is mixed with the SM top quark after the electroweak symmetry breaking, which leads to the decays modes $t_{p} \rightarrow b W$ and $t_{p} \rightarrow t Z$. On the other hand, the decay into th comes from the Yukawa interaction of the top partner and a dimension-five oparator. If some 
strong dynamics is the origin of new physics, such as in the little higgs model, the dimension-five operator can be sizable and the decay $t_{p} \rightarrow$ th is enhanced.

The top partner decaying into $b W$ and $t Z$ have already been searched at the LHC experiment. For example, the CMS collaboration has searched for the process, $p p \rightarrow t_{p} \bar{t}_{p} X$ followed by the decay $t_{p} \rightarrow b W$, in a b-jet of high $p_{T}$ and lepton(s) channels with the integrated luminosity of $4.7 \mathrm{fb}^{-1}$. They have put a limit $m_{t p}>$ $560 \mathrm{GeV}$ with $\operatorname{Br}\left(t_{p} \rightarrow b W\right)=1$ [10], where $m_{t p}$ is the top partner mass. The CMS collaboration has also searched for another process, $p p \rightarrow t_{p} \bar{t}_{p} X$ followed by the decay $t_{p} \rightarrow t Z$, in multi-jet and three-lepton channel with $1.1 \mathrm{fb}^{-1}$ data. They have put a limit $m_{t p}>475 \mathrm{GeV}$ with $\operatorname{Br}\left(t_{p} \rightarrow t Z\right)=1[11$.

Experimental results based on $t_{p} \rightarrow$ th have not been reported yet, although this is very important to know the origin of the top partner. The recent LHC data indicates the higgs mass of about $125 \mathrm{GeV}$ [5, 6], and the higgs boson mostly decays into $b \bar{b}$ in this mass region. Since the decay mode $t_{p} \rightarrow$ th produces three bottom quarks in the presence of a such light higgs boson, multi-b-jet channels are expected to be sensitive for the detection of the top partner. In order to quantitatively investigate this expectation, we perform simulations including detector effects, and estimate the sensitivity of the LHC experiment to the top partner using multi-b-jet channels at the center of mass energy of $8 \mathrm{TeV}$. For comparison, we also estimate the sensitivity of the LHC experiment in the one b-jet and one lepton channel which has been adopted in the ATLAS and CMS collaborations.

There are previous studies that considerer the decay $t_{p} \rightarrow$ th [12, 13, 14]. Especially, one of them investigates the top partner assuming the light higgs boson based on more than three b-jets at the $14 \mathrm{TeV}$ run of the LHC experiment [12], and another one studies the $t_{p} \rightarrow t h$ followed by the decay of $h \rightarrow \gamma \gamma$ and $Z Z[13$ at 7 $\mathrm{TeV}$ and $14 \mathrm{TeV}$.

We start with, in the next section, the effective action describing the top partner in order to make the discussion as general as possible. In Section 3, we describe our simulation setup and perform the analyses in the multi-b-jet channels, and show the sensitivity of the LHC experiment at the $8 \mathrm{TeV}$ run. According to results obtained in these analyses, we discuss some implications to new physics models beyond the SM in Section 4. Section 5 is devoted to summary of our discussions. 


\section{Effective action for the top partner}

In order for our discussion to be as general as possible, we give an effective action of the top partners up to dimension-five operators. It is shown that a dimensionfive operator actually takes an important role to enhance the branching fraction of the decay mode $t_{p} \rightarrow$ th which leads multiple b-jets at the LHC experiment. For more detail of this effective action, see Ref. [3]. Since the top partner has quantum numbers of $(\mathbf{3}, \mathbf{1}, 2 / 3)$ under the $\mathrm{SM}$ gauge groups, $\mathrm{SU}(3)_{c} \times \mathrm{SU}(2)_{L} \times \mathrm{U}(1)_{Y}$, its interactions with higgs boson and third generation quarks of the SM are given by

$$
\mathcal{L}_{\text {eff }}=-m_{U} \bar{U}_{L} U_{R}-y_{3} \bar{Q}_{3 L} H^{c} u_{3 R}-y_{U} \bar{Q}_{3 L} H^{c} U_{R}-(\lambda / \Lambda) \bar{U}_{L} u_{3 R}|H|^{2}+\text { h.c. }
$$

where $H, Q_{3 L}$, and $u_{3 R}$ are higgs doublet, third generation left- and right-handed quarks, respectively, while $U_{L}$ and $U_{R}$ are left- and right-handed components of the top partner. The superscript ' $c$ ' denotes charge conjugation, and $\Lambda$ in front of the dimension-five operator, $\bar{U}_{L} u_{3 R}|H|^{2}$, is the cutoff scale, where the above effective action can be applied to describe physics below this scale. The other dimension-five operators such as $\left(\bar{U}_{L} U_{R}|H|^{2}\right) / \Lambda$ are irrelevant to our discussion. The top partner has the QCD interaction in addition to those in Eq.(1). All the parameters in the effective action can be real by appropriate redefinitions of the fields. Note that there are only three free parameters because the top quark mass $m_{t}$ has already been measured. The use of this effective action is particularly useful for the little higgs model, because the top partner is nothing but a new particle which is introduced to cancel the quadratically divergent correction to the higgs mass term from the top loop diagram, and therefore the top partner mass should be lighter than the other new particles. The effective action is valid only when the other new particles are heavy enough compared to the top partner.

After the electroweak symmetry is broken down, left- and right-handed components of the top partner $\left(U_{L}\right.$ and $\left.U_{R}\right)$ are mixed with those of the SM top quark,

$$
\left(\begin{array}{c}
t_{L} \\
t_{p_{L}}
\end{array}\right)=\left(\begin{array}{cc}
\cos \theta_{t L} & -\sin \theta_{t L} \\
\sin \theta_{t L} & \cos \theta_{t L}
\end{array}\right)\left(\begin{array}{c}
u_{3 L} \\
U_{L}
\end{array}\right), \quad\left(\begin{array}{c}
t_{R} \\
t_{p_{R}}
\end{array}\right)=\left(\begin{array}{cc}
\cos \theta_{t R} & -\sin \theta_{t R} \\
\sin \theta_{t R} & \cos \theta_{t R}
\end{array}\right)\left(\begin{array}{c}
u_{3 R} \\
U_{R}
\end{array}\right),
$$

where these mixing matrices diagonalize the mass matrix of the top partner and the top quark, giving their mass eigenvalues $m_{t p}$ and $m_{t}$. In the following, we take $m_{t p}$, $m_{t}$, and $\sin \theta_{t L}$, and $\sin \theta_{t R}$ as model parameters instead of $m_{U}, y_{3}, y_{U}$, and $\lambda / \Lambda$ which are originally defining the effective action. With $s_{t L}\left(c_{t L}\right)$ and $s_{t R}\left(c_{t R}\right)$ being 
$\sin \theta_{t L}\left(\cos \theta_{t L}\right)$ and $\sin \theta_{t R}\left(\cos \theta_{t R}\right)$, respectively, the original parameters are given

$$
\begin{aligned}
m_{U} & =s_{t R} s_{t L} m_{t}-c_{t R} c_{t L} m_{t p} \\
y_{3} & =(\sqrt{2} / v)\left(c_{t R} c_{t L} m_{t}+s_{t R} s_{t L} m_{t p}\right), \\
y_{U} & =(\sqrt{2} / v)\left(-s_{t R} c_{t L} m_{t}+c_{t R} s_{t L} m_{t p}\right), \\
\lambda / \Lambda & =\left(2 / v^{2}\right)\left(-c_{t R} s_{t L} m_{t}+s_{t R} c_{t L} m_{t p}\right),
\end{aligned}
$$

where $v$ is the vacuum expectation value of the higgs field. All physical quantities related to the LHC signal of the top partner are obtained with the use of this parameterization as well as gauge interactions of top partner and top quark.

In Fig 1, the branching fraction of the decay of the top partner is depicted as a function of $\sin \theta_{t R}$ with $\sin \theta_{t L}=0.1$ and $m_{t_{p}}=500 \mathrm{GeV}$. As can be seen in Section 4 , the left-handed mixing angle $\sin \theta_{t L}$ is severely constrained by the electroweak precision measurement and thus should be small enough. Since branching fractions of decay modes $t_{p} \rightarrow b W$ and $t_{p} \rightarrow t Z$ are proportional to $\sin ^{2} \theta_{t L}$, the decay mode $t_{p} \rightarrow$ th has a large branching fraction when $\sin \theta_{t R}$ is sizable. Since the first term of Eq.([6) is negligible compared to the second term, the right-handed mixing angle $\sin \theta_{t R}$ depends strongly on the coefficient of the dimension-five operator $\lambda / \Lambda$. In the little higgs model, $\Lambda / \lambda$ is of the order of the top partner mass $m_{t p}$, and, as a result, $\sin \theta_{t R}$ can be as large as 0.1 . In such a case, the branching fraction of the decay mode $t_{p} \rightarrow$ th is enhanced and the use of the multi-b-jet channel becomes efficient to search for the top partner. When the scale $\Lambda$ is large enough as in the most cases of weak-interacting new physics models, the angle $\sin \theta_{t R}$ is almost zero.

\section{Multi-b-jet channel}

In this section, we study the top partner signature in multi-b-jet channels and the SM backgrounds. After describing our simulation framework and selection-criteria to reduce the backgrounds, we present the sensitivity of the LHC experiment to the top partner signal in not only multi-b-jet channels but also the channel used in previous analyses of the ATLAS and CMS collaborations. The center of mass energy is set to be $8 \mathrm{TeV}$ and higgs mass is $120 \mathrm{GeV}$ throughout this article. 


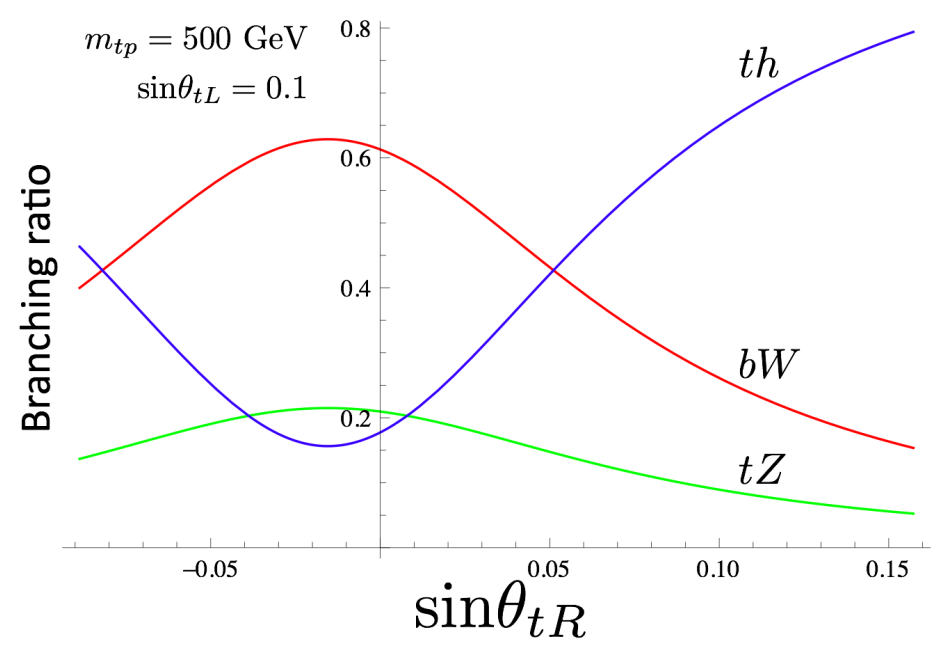

Figure 1: The branching fraction of the decay of the top partner $\left(t_{p} \rightarrow b W, t Z\right.$, and $\left.t h\right)$ as a function of $\sin \theta_{t R}$ with $\sin \theta_{t L}=0.1$ and $m_{t_{p}}=500 \mathrm{GeV}$.

\subsection{Simulation framework}

Using the Feynrules [15] package, we first implement the interactions of $t_{p}$ into MadGraph5 [16] based on the effective action. Parton level events are interfaced to PYTHIA6.420 [17] for parton-showering and hadronization, and Delphes1.9 [18] is used to simulate detector effects. We set appropriate resolution-parameters for the detector simulation based on the ATLAS detector performance [19].

We adopt the method to reconstruct objects such as isolated, central leptons and jets according to the strategy of new physics searches at the ATLAS experiment [20]. Jet candidates are reconstructed using the anti- $k_{T}$ algorithm [21] implemented in Delphes1.9 with the radius parameter $R=0.4$. The jet candidates are required to have the transverse momentum $p_{T}>20 \mathrm{GeV}$ and the pseudo rapidity $|\eta|<$ 2.8. Electron and muon candidates are identified via the generator-data assuming $100 \%$ efficiency. The electron(muon) candidates are required to have $p_{T}>20(10)$ $\mathrm{GeV}$ and $|\eta|<2.47(2.4)$. After these pre-selections, overlaps between the electron candidates and the jet candidates are removed. The jet candidates are discarded if their distances $\Delta R=\sqrt{\Delta \eta^{2}+\Delta \phi^{2}}$ to any electron candidates are less than 0.2 where $\Delta \eta(\Delta \phi)$ is the difference of the pseudo rapidity (azimuthal angle) between the jet candidate and the electron candidate. The remaining jet candidates are called "jets". For each jets, the electron candidates with $0.2<\Delta R<0.4$ from jets are removed. Furthermore, isolation criteria are imposed. The electron candidates are 


\begin{tabular}{|c|cccccccc|}
\hline Mass $(\mathrm{GeV})$ & 400 & 450 & 500 & 550 & 600 & 650 & 700 & 750 \\
\hline Cross section $(\mathrm{pb})$ & 2.0 & 0.99 & 0.51 & 0.27 & 0.15 & 0.086 & 0.051 & 0.030 \\
\hline
\end{tabular}

Table 1: Cross sections of $p p \rightarrow t_{p} \bar{t}_{p}+X$ with several choices of the top partner mass.

removed if the scalar sums of the transverse momentum of tracks within a cone size of $\Delta R=0.2$ around the electron candidates exceed $10 \%$ of the electron candidates. The muon candidates are removed if the scalar sums of the transverse momentum of tracks within a cone size of $\Delta R=0.2$ around the muon candidates exceed $1.8 \mathrm{GeV}$. The remaining electron(muon) candidates are called "electrons(muons)". We also take a calibrated efficiency and a mis-tagging rate for the b-tagging obtained by the SVO50 method [22]. These results are well fitted by

$$
\begin{aligned}
(\mathrm{b}-\mathrm{tag} \text { efficiency }) & =0.6 \tanh \left(p_{T} / 36 \mathrm{GeV}\right) \times(1.02-0.02|\eta|), \\
(\text { mistag rate for light-jet }) & =0.001+0.00005\left(p_{T} / 1 \mathrm{GeV}\right),
\end{aligned}
$$

where $p_{T}$ and $\eta$ are transverse momentum and pseudo-rapidity of the jet, respectively. On the other hand, we use the value of 0.14 as the mistag rate for $c$-jet, which is obtained by the method of the secondary vertex [23]. We find our detector simulation reproduces the ATLAS data involving a b-jet, leptons and missing energy within 10\% accuracy. The comparison with the ATLAS data is presented in appendix A.

The top partner can be copiously produced unless it is too heavy. The dominant process to search for the top partner is the pair production, $p p \rightarrow t_{p} \bar{t}_{p}+X$. The production cross section obtained by HATHOR 25] is shown in Table 1. From the effective action Eq.(1), the top partner has three decay modes; $t_{p} \rightarrow b W, t Z$, and th. In the previous study, the top partner has been searched for in one b-jet and one lepton channel aiming for the $t_{p} \rightarrow b W$ decay. In this article, we study the decay mode $t_{p} \rightarrow$ th which is sensitive to the cutoff scale of the little higgs model, as mentioned in Section 2. In order to search for the decay, the signature with multiple b-jets is more important since one $t_{p}$-decay can produce three b-jets.

There are several background processes against the signal, which are listed in Table 2. In order to generate the $t \bar{t}+$ jets events, we have considered processes associated with up to two additional partons $(t \bar{t}, t \bar{t} j$, and $t \bar{t} j j)$. These events are matched after the parton-showering using the MLM matching scheme implemented in MadGraph. For the generation of the $W+$ jets events, the $W$ boson is forced to decay leptonically and is associated with up to three additional partons, where 


\begin{tabular}{c|cc} 
& Remarks & Cross section $(\mathrm{pb})$ \\
\hline$t \bar{t}+$ jets & up to 2 jets & 204 \\
$W+$ jets & up to 3 jets & 320 \\
& $\left(W \rightarrow l \nu, \quad p_{T-1 \text { st,2nd } \mathrm{j}}>70,40 \mathrm{GeV}\right)$ & \\
$t \bar{t} b \bar{b}$ & $\left(p_{T-1 \text { st b }}>30 \mathrm{GeV}\right)$ & 1.6 \\
\hline
\end{tabular}

Table 2: Background processes against the signal of the top partner.

the first and second partons are required to have $p_{T}>70 \mathrm{GeV}$ and $40 \mathrm{GeV}$, respectively. For $t \bar{t} b \bar{b}$ events, the first b-parton is required to have $p_{T}>30 \mathrm{GeV}$. The background conditions as well as their cross sections are also listed in the second and third columns of Table 2. The total cross section of $t \bar{t}+$ jets is normalized to be 204 pb, which is again obtained by HATHOR. For the others, we use the matched cross sections obtained from the MadGraph-PYTHIA package. There are other possible backgrounds such as $Z+$ jets, $W b \bar{b}+$ jets, $Z b \bar{b}+$ jets and $b \bar{b} b \bar{b}$, but we have confirmed those are negligible in our analysis.

\subsection{Event selections}

\subsubsection{Single-b-jet channel: 1 lepton $+\geq 1 \mathrm{~b}$-jet}

The pair production of top partners followed by the decays into two bottom quarks and two $W$ bosons has been searched for in the CMS collaboration [10]. The onelepton channel, $t_{p} \bar{t}_{p} \rightarrow b W^{+} \bar{b} W^{-} \rightarrow b \bar{b} l^{ \pm} \nu j j$, currently gives the most stringent bound on the $t_{p} \bar{t}_{p}$ production. We therefore impose the following selection-criteria in order to evaluate the LHC-sensitivity to the signal,

1. There is only one lepton which is required to have $p_{T}>30 \mathrm{GeV}$.

2. Transverse missing energy $\mathbb{E}_{T}$ should be larger than $20 \mathrm{GeV}$.

3. There are more than three jets, and at least one of them is b-tagged.

4. Leading four jets are required to have $p_{T}>80,50,30$, and $30 \mathrm{GeV}$.

Energetic b-jets are expeted from any decay pettern of $t_{p} \bar{t}_{p}$. The distribution of invariant mass $M_{b l \nu}$ is very useful to separate signal events from those of the SM backgrounds mostly from top quarks. Here, the invariant mass $M_{b l \nu}$ is reconstructed from the leading b-jet, a charged lepton detected, and missing transverse momentum $P_{\text {Tmiss }}$. The transverse momentum of the neutrino is identified with $P_{\text {Tmiss }}$, while 
the longitudinal one is determined so that the $W$ boson mass is reproduced from the lepton and the neutrino. In order to deal with the two-fold ambiguity, which appears in the determination of the longitudinal momentum, we always take the smaller $M_{b l \nu}$. If there is no solution of the longitudinal momentum that satisfies $\left(p_{\text {lepton }}+p_{\text {miss }}\right)^{2}=$ $m_{W}^{2}$, we take the momentum so that it gives the minimal $M_{b l \nu}$. We then require following three more selection-criteria to generated signal and background events,

5. The leading b-jet is required to have $p_{T}>260 \mathrm{GeV}$.

6. The invariant mass $M_{\mathrm{bl} \nu}$ should be larger than $400 \mathrm{GeV}$

7. The effective mass $M_{\text {eff }}$ should be larger than $1000 \mathrm{GeV}$, where the effective mass in the above criteria is defined by $M_{\text {eff }}=p_{T \text {-lepton }}+\mathbb{E}_{T}+$ $\sum_{i=1}^{\min \left(5, N_{\mathrm{jets}}\right)} p_{T \text {-ith } \mathrm{j}}$. Here, $N_{\text {jets }}$ is the number of the jets in the events. These criteria enable us to reduce $t \bar{t}+$ jets background significantly.

Distributions of $p_{T-1 \mathrm{stb}}$ (the transverse momentum of the leading b-jet), $M_{\mathrm{bl} \nu}$, and $M_{\text {eff }}$ after applying kinematical cuts 1-4 are plotted in Fig, 2 with $m_{t p}=500$ $\mathrm{GeV}$. Distributions of $t_{p} \bar{t}_{p} \rightarrow b W b W, b W t h$ and thth are shown separately assuming all $t_{p} \bar{t}_{p}$-decays in each mode. The distribution of the $t \bar{t}+$ jets events is also plotted in the same figure. The $p_{T}$-distribution of b-jet for $t_{p} \bar{t}_{p} \rightarrow b W b W$ is highest among all the distributions and has a clear peak at $M_{b l \nu} \sim m_{t p}$. The separation between signal and background is the worst for the $t_{p} \bar{t}_{p} \rightarrow$ thth events.

We present the cut flows of both signal and background events in Table 3. Numbers of the signal and background events are normalized to those corresponding to $15 \mathrm{fb}^{-1}$, which are shown in the third low. In the last low of Table 3, we give acceptances after all the kinematical cuts imposed. Acceptances of the signal events for various masses of the top partner are also found in Table 4 , The $t_{p} \bar{t}_{p} \rightarrow b W t h$ and thth events are found to decrease after applying kinematical cuts 5 and 6 . The contribution of the $t_{p} \bar{t}_{p} \rightarrow b W t h$ channel is larger when the top partner mass is heavier, because it is easier to satisfy kinematical cuts on $p_{T}$.

For comparison, in appendix $\mathrm{B}$, we also show the results based on the CMSanalysis method described in Ref.[26]. Our estimate gives a better result than that based on the CMS analysis.

\subsubsection{Two-b-jet channles: 1 lepton $+\geq 2$ b-jets}

We next consider the two-b-jet channels to search for the top partner. Both decay modes of the top partner, $t_{p} \rightarrow b W$ and $t_{p} \rightarrow t h$, are expected to contribute to signal 

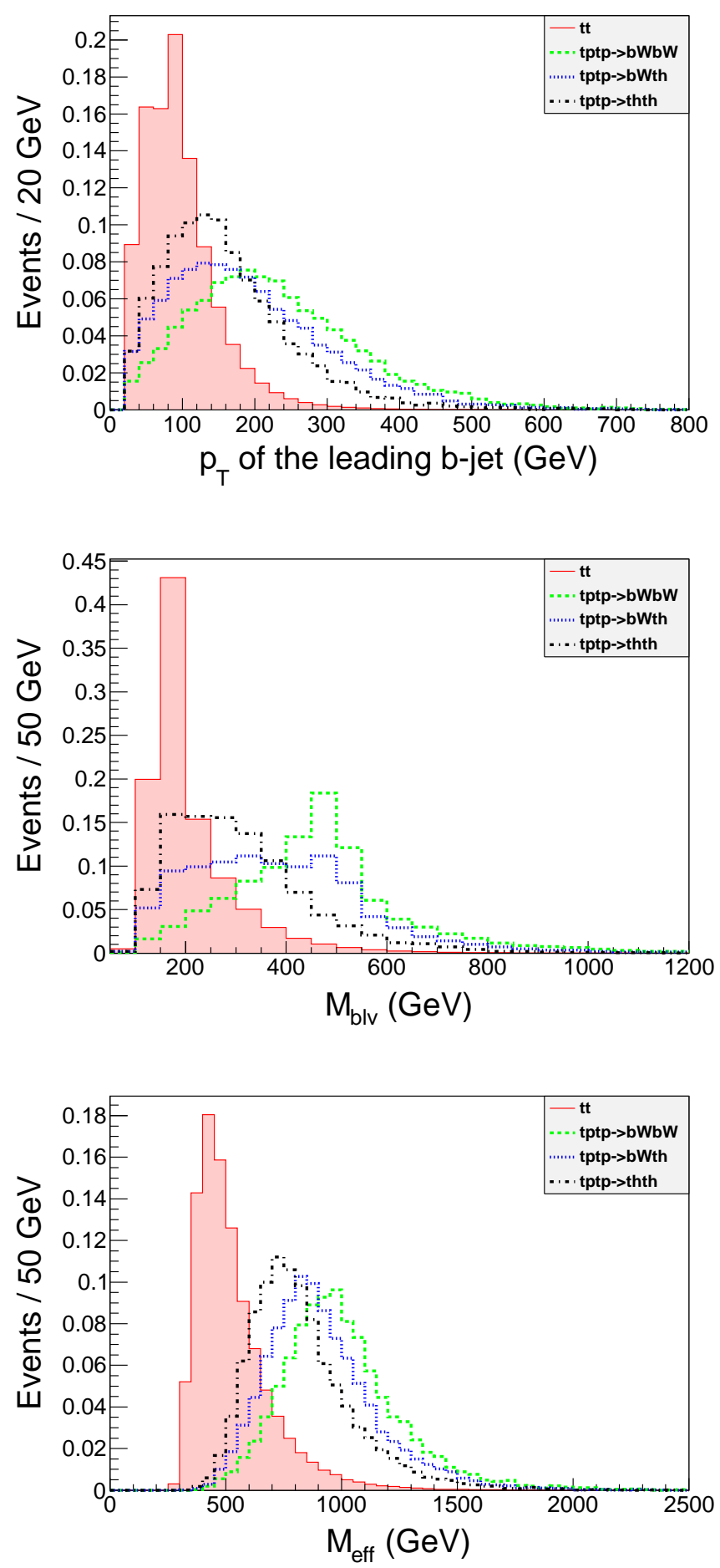

Figure 2: Distributions of $p_{T \text {-1st b }}$ (the transverse momentum of the leading b-jet), $M_{\mathrm{bl} \nu}$, and $M_{\text {eff }}$ after applying kinematical cuts $1-4$ in the analysis of the ' 1 lepton $+\geq 1 \mathrm{~b}$ jet' channel. The mass of the top partner is $m_{t p}=500 \mathrm{GeV}$. Distributions of $t_{p} \bar{t}_{p} \rightarrow$ $b W b W, b W t h$ and thth are shown separately assuming all $t_{p} \bar{t}_{p}$-decays in each mode. The distributions are normalized so that their integrated values become unity. 


\begin{tabular}{c|ccccc} 
& $t \bar{t}$ & $W+$ jets & $T \bar{T} \rightarrow b W b W$ & $T \bar{T} \rightarrow b W t h$ & $T \bar{T} \rightarrow$ thth \\
\hline Generated & 6163292 & 809561 & 60000 & 60000 & 60000 \\
Without cuts & 3060000 & 4800000 & 7650 & 7650 & 7650 \\
Cuts 1-3 & 282861 & 16756 & 1336 & 1652 & 1693 \\
Cut 4 & 133099 & 6392 & 1160 & 1555 & 1654 \\
Cut 5 & 1872 & 415 & 405 & 374 & 215 \\
Cut 6 & 1025 & 320 & 351 & 287 & 125 \\
Cut 7 & 387 & 160 & 237 & 164 & 75 \\
\hline Acceptance & 0.00013 & 0.000033 & 0.031 & 0.021 & 0.0099 \\
\hline
\end{tabular}

Table 3: Cut flows of the signal and background events in the ' 1 lepton $+\geq 1$ b-jet' channel. The mass of the top partner is $m_{t p}=500 \mathrm{GeV}$. Results of $t_{p} \bar{t}_{p} \rightarrow b W b W, b W t h$ and thth are shown separately assuming all $t_{p} \bar{t}_{p^{-}}$-decays in each mode.

\begin{tabular}{c|cccccccc} 
Mass $(\mathrm{GeV})$ & 400 & 450 & 500 & 550 & 600 & 650 & 700 & 750 \\
\hline$T \bar{T} \rightarrow b W b W$ & 0.0096 & 0.018 & 0.031 & 0.047 & 0.062 & 0.074 & 0.084 & 0.092 \\
$T \bar{T} \rightarrow b W t h$ & 0.0067 & 0.013 & 0.021 & 0.034 & 0.048 & 0.063 & 0.076 & 0.090 \\
$T \bar{T} \rightarrow$ thth & 0.0030 & 0.0050 & 0.0098 & 0.016 & 0.026 & 0.036 & 0.049 & 0.059 \\
\hline
\end{tabular}

Table 4: Acceptances of the signal events in the ' 1 lepton $+\geq 1$ b-jet' channel with several choices of the top partner mass. Results of $t_{p} \bar{t}_{p} \rightarrow b W b W, b W t h$ and thth are shown separately assuming all $t_{p} \bar{t}_{p}$-decays in each mode.

events in this channel. On the other hand, certain amounts of background events are also expected from $t \bar{t}+$ jets processes. As in the case of the single-b-jet channel, we also focus on the two-b-jet channels associated with semi-leptonic decays of two $W$ bosons to reduce the SM backgrounds,

$$
P P \rightarrow t_{p} \bar{t}_{p} \rightarrow b W b W, b W t h, \text { thth } \rightarrow W W+\text { b-jets } \rightarrow l \nu j j+\text { b-jets. }
$$

Therefore, in the analysis of this channel, an efficient selection-criteria turns out to be essentially the same as that of the single-b-jet analysis, except for the number of b-jets in order to extract the signal events with multiple b-jets. With the use of exactly the same method for the reconstruction of the invariant mass $M_{b l \nu}$ adopted in the previous single-b-jet analysis, we impose the following selection-criteria

1. There is only one lepton which is required to have $p_{T}>30 \mathrm{GeV}$.

2. Transverse missing energy $\mathbb{E}_{T}$ should be larger than $20 \mathrm{GeV}$. 


\begin{tabular}{c|ccccc} 
& $t \bar{t}$ & $t \bar{t} b \bar{b}$ & $T \bar{T} \rightarrow b W b W$ & $T \bar{T} \rightarrow b W t h$ & $T \bar{T} \rightarrow$ thth \\
\hline Generated & 6163292 & 90000 & 60000 & 60000 & 60000 \\
Without cuts & 3060000 & 24000 & 7650 & 7650 & 7650 \\
Cuts 1-3 & 56143 & 1677 & 413 & 936 & 1227 \\
Cut 4 & 317 & 17 & 103 & 100 & 79 \\
Cut 5 & 209 & 14 & 100 & 92 & 67 \\
Cut 6 & 33 & 3 & 37 & 30 & 20 \\
\hline Acceptance & 0.000011 & 0.00011 & 0.0049 & 0.0040 & 0.0026 \\
\hline
\end{tabular}

Table 5: Cut flows of signal and background events in the analysis of the " 1 lepton + $\geq 2 \mathrm{~b}$-jets' channel. The mass of the top partner is $m_{t_{p}}=500 \mathrm{GeV}$. Results of $t_{p} \bar{t}_{p} \rightarrow$ $b W b W, b W t h$ and thth are shown separately assuming all $t_{p} \bar{t}_{p}$-decays in each mode.

3. There are more than four jets, and at least two of them are b-tagged.

4. The b-jets are required to have $p_{T}>200,180 \mathrm{GeV}$.

5. The invariant mass $M_{b l \nu}$ should be larger than $250 \mathrm{GeV}$.

6. The effective mass $M_{\text {eff }}$ should be larger than $1200 \mathrm{GeV}$.

Distributions of $M_{\mathrm{eff}}, M_{b l \nu}$, and transverse momenta of the first and second bjets after applying kinematical cuts 1-3 are plotted in Fig.3. As in the case of the previous single-b-jet analysis, the mass of the top partner for signal events is again $m_{t_{p}}=500 \mathrm{GeV}$, and we concider decay patterns of $t_{p} \bar{t}_{p} \rightarrow b W b W, b W t h$, and thth. The distribution of $t \bar{t}+$ jets events is plotted in the same figure. The cut flows of the signal events and the main background events $(t \bar{t}+$ jets and $t \bar{t} b \bar{b})$ are shown in Table 5 . Numbers of events for signals and backgrounds in the third low correspond to those with the integrated luminosity of $15 \mathrm{fb}^{-1}$, while their acceptances after applying all kinematical cuts 1-6 are shown in the last low. The acceptances in each decay pattern of $t_{p} \bar{t}_{p}$ are found in Table 6 .

\subsubsection{Three-b-jet channels: 1 lepton $+\geq 3$ b-jets}

We finally consider the three-b-jet channels to search for the top partner. This channel relies on the decay mode $t_{p} \rightarrow t h$. Since the higgs mass is expected to be about 120-130 GeV as strongly suggested by recent data of the ATLAS [5] and the CMS [6] collaborations, the higgs boson decays mainly into two bottom quarks, and, as a result, the decay of the top partner produces three bottom quarks. Several 

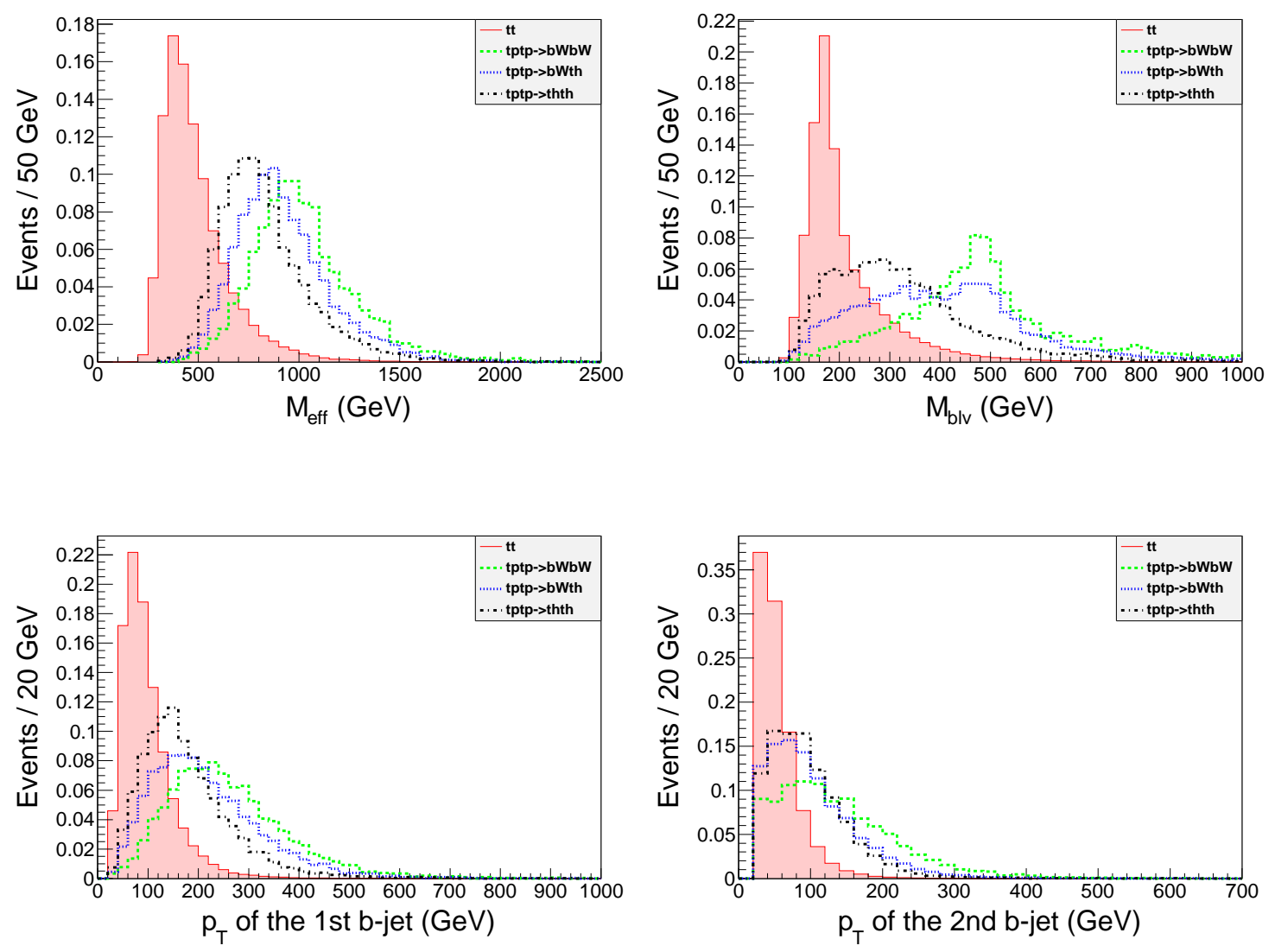

Figure 3: Distributions of $M_{\mathrm{eff}}, M_{b l \nu}$, and transverse momenta of the first and second bjets for signal and background $(t \bar{t}+$ jets $)$ events after applying the cuts $1-3$ in the analysis of the ' 1 lepton $+\geq 2$ b-jets' channel. The mass of the top partner is $m_{t p}=500 \mathrm{GeV}$. Distributions of $t_{p} \bar{t}_{p} \rightarrow b W b W, b W t h$ and thth are shown separately assuming all $t_{p} \bar{t}_{p^{-}}$ decays in each mode. The distributions are normalized so that their integrated values become unity. 


\begin{tabular}{c|cccccccc} 
Mass $(\mathrm{GeV})$ & 400 & 450 & 500 & 550 & 600 & 650 & 700 & 750 \\
\hline$T \bar{T} \rightarrow b W b W$ & 0.0015 & 0.0026 & 0.0049 & 0.0075 & 0.012 & 0.016 & 0.020 & 0.024 \\
$T \bar{T} \rightarrow b W t h$ & 0.0010 & 0.0023 & 0.0040 & 0.0065 & 0.011 & 0.016 & 0.022 & 0.028 \\
$T \bar{T} \rightarrow$ thth & 0.00077 & 0.0015 & 0.0026 & 0.0040 & 0.0064 & 0.011 & 0.015 & 0.022 \\
\hline
\end{tabular}

Table 6: Acceptances of signal events in the ' 1 lepton $+\geq 2$ b-jets' channel with several choices of the top partner mass. Results of $t_{p} \bar{t}_{p} \rightarrow b W b W, b W t h$ and thth are shown separately assuming all $t_{p} \bar{t}_{p}$-decays in each mode.

three-b-jet channels following the pair production of the top partner are, in fact, available. Among those, in order to reduce SM backgrounds efficiently, we again focus on the channel associated with semi-leptonic decays of two $W$ bosons,

$$
\begin{aligned}
& t_{p} \bar{t}_{p} \rightarrow b W t h \rightarrow(b W)(b W b b) \rightarrow b b b b l \nu j j, \\
& t_{p} \bar{t}_{p} \rightarrow t h t h \rightarrow(b W b b)(b W b b) \rightarrow b b b b b b l \nu j j .
\end{aligned}
$$

As in cases of single-b-jet and two-b-jet channels, we impose following selectioncriteria with adopting the same method to reconstruct the invariant mass $M_{b l \nu}$,

1. There is only one lepton which is required to have $p_{T}>30 \mathrm{GeV}$.

2. Transverse missing energy $\mathbb{E}_{T}$ should be larger than $20 \mathrm{GeV}$.

3. There are more than four jets, and at least three of them are b-tagged.

4. The b-tagged jets are required to have $p_{T}>140,80,80 \mathrm{GeV}$.

5. The invariant mass $M_{b l \nu}$ should be larger than $250 \mathrm{GeV}$.

6. The effective mass $M_{\text {eff }}$ should be larger than $1000 \mathrm{GeV}$.

Distributions of $M_{\mathrm{eff}}, M_{b l \nu}$, and transverse momenta of the first to third b-jets after applying kinematical cuts 1-3 are plotted in Fig, 4. As in the cases of singleb-jet and two-b-jet analyses, the mass of the top partner for signal events is $m_{t_{p}}=$ $500 \mathrm{GeV}$, and we concider decay patterns of $t_{p} \bar{t}_{p} \rightarrow b W b W, b W t h$, and thth. The distribution of $t \bar{t}+$ jets events is plotted in the same figure. The cut flows of the signal events and the main background events $(t \bar{t}+$ jets and $t \bar{t} b \bar{b})$ are shown in Table 7. Numbers of events for signals and backgrounds in the third low correspond to those with the integrated luminosity of $15 \mathrm{fb}^{-1}$, while their acceptances after applying all kinematical cuts 1-6 are shown in the last low. The acceptances in each decay pattern of $t_{p} \bar{t}_{p}$ are found in Table 8 . 

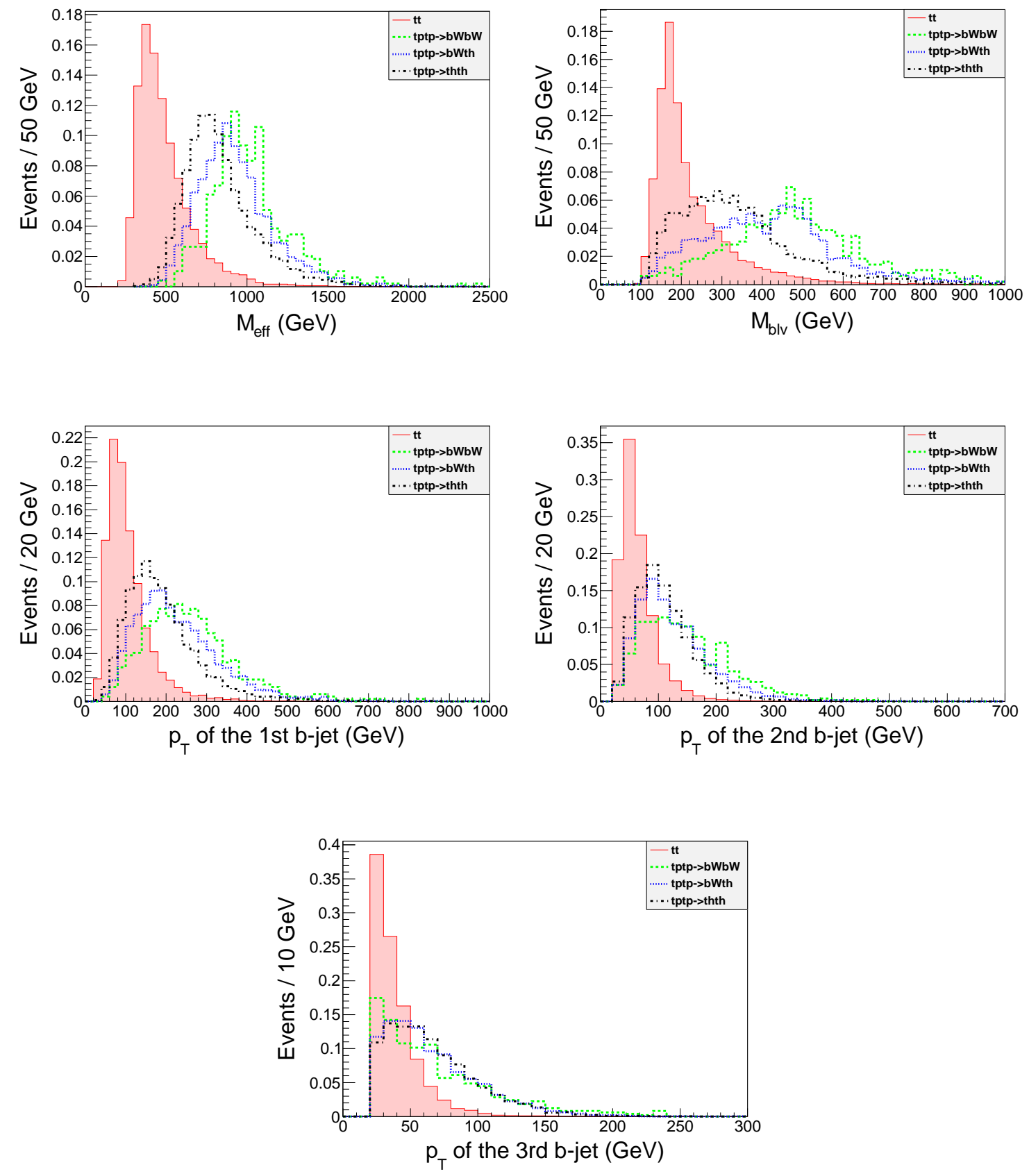

Figure 4: Distributions of $M_{\text {eff }}, M_{b l \nu}$, and transverse momenta of the first to third b-jets for signal and background ( $t \bar{t}+$ jets) events after applying the cuts $1-3$ in the analysis of the ' 1 lepton $+\geq 3$ b-jets' channel. The mass of the top partner is $m_{t p}=500 \mathrm{GeV}$. Distributions of $t_{p} \bar{t}_{p} \rightarrow b W b W, b W t h$ and thth are shown separately assuming all $t_{p} \bar{t}_{p}$-decays in each mode. The distributions are normalized so that their integrated values become unity. 


\begin{tabular}{c|ccccc} 
& $t \bar{t}$ & $t \bar{t} b \bar{b}$ & $T \bar{T} \rightarrow b W b W$ & $T \bar{T} \rightarrow b W t h$ & $T \bar{T} \rightarrow$ thth \\
\hline Generated & 6163292 & 90000 & 60000 & 60000 & 60000 \\
Without cuts & 3060000 & 24000 & 7650 & 7650 & 7650 \\
Cuts 1-3 & 5328 & 558 & 63 & 380 & 686 \\
Cut 4 & 123 & 26 & 19 & 99 & 170 \\
Cut 5 & 78 & 18 & 17 & 86 & 135 \\
Cut 6 & 15 & 4 & 11 & 40 & 45 \\
\hline Acceptance & 0.0000050 & 0.00016 & 0.0014 & 0.0052 & 0.0059 \\
\hline
\end{tabular}

Table 7: Cut flows of signal and background events in the analysis of the ' 1 lepton + $\geq 3$ b-jets' channel. The mass of the top partner is $m_{t p}=500 \mathrm{GeV}$. Results of $t_{p} \bar{t}_{p} \rightarrow$ $b W b W, b W t h$ and thth are shown separately assuming all $t_{p} \bar{t}_{p}$-decays in each mode.

\begin{tabular}{c|cccccccc} 
Mass $(\mathrm{GeV})$ & 400 & 450 & 500 & 550 & 600 & 650 & 700 & 750 \\
\hline$T \bar{T} \rightarrow b W b W$ & 0.00070 & 0.0014 & 0.0014 & 0.0022 & 0.0029 & 0.0034 & 0.0039 & 0.0042 \\
$T \bar{T} \rightarrow b W t h$ & 0.0018 & 0.0035 & 0.0052 & 0.0098 & 0.013 & 0.016 & 0.019 & 0.022 \\
$T \bar{T} \rightarrow t h t h$ & 0.0014 & 0.0034 & 0.0059 & 0.0092 & 0.015 & 0.021 & 0.026 & 0.031 \\
\hline
\end{tabular}

Table 8: Acceptances of signal events in the ' 1 lepton $+\geq 3$ b-jets' channel with several choices of the top partner mass. Results of $t_{p} \bar{t}_{p} \rightarrow b W b W, b W t h$ and thth are shown separately assuming all $t_{p} \bar{t}_{p}$-decays in each mode.

\subsection{LHC-sensitivity to the top partner at $8 \mathrm{TeV}$}

We are now at the position to discuss the LHC-sensitivity to the top partner. In the following analysis, the systematic uncertainty of the SM backgrounds is simply set to be $20 \%$ in all of these channels. This value is based on that obtained in the CMS experiment [26] for the 1 lepton $+\geq 1$ b-jet channel. Exclusion region is defined as the one which is excluded at 95\% C.L. if the observed number of the events is equal to the expected number of the SM background events. Discovery region is the one where the expected number of the signal and the SM background events deviates from the expected number of the SM background events at $5 \sigma$ level. For this calculation, we utilized the method reviewed in Ref.[27].

Based on the SM backgrounds evaluated in the previous subsection, expected exclusion-limits on signal cross sections in the single-, two-, and three-b-jet channels 
at $95 \%$ C.L. when no signal is detected turn out to be

$$
(\text { Cross section } \times \text { Acceptance })>\left\{\begin{aligned}
4.4 \mathrm{fb}: & 1 \text { lepton }+\geq 1 \text { b-jet } \\
1.2 \mathrm{fb}: & 1 \text { lepton }+\geq 2 \text { b-jets } \\
0.92 \mathrm{fb}: & 1 \text { lepton }+\geq 3 \text { b-jets. }
\end{aligned}\right.
$$

For the integrate luminosity of $15 \mathrm{fb}^{-1}$, the regions which would be constrained by the analysis of the single-b-jet channel (1 lepton $+\geq 1$ b-jet), two-b-jet channels (1 lepton $+\geq 2$ b-jets), and three-b-jet channels ( 1 lepton $+\geq 3$ b-jets) are plotted in upper, middle, and lower panels of Fig,5, respectively. The regions are depicted on the plane of $\operatorname{Br}\left(t_{p} \rightarrow b W\right)$ and $\operatorname{Br}\left(t_{p} \rightarrow t h\right)$ for various values of the top partner mass. The single- and two-b-jet channels give stringent constraints on $m_{t p}$ when the top partner decays dominantly into $b W$, while the three-b-jet channels constrains the top partner when $\operatorname{Br}\left(t_{p} \rightarrow t h\right)$ is large. The LHC experiment has a capability to exclude $m_{t p}$ less than 750 (650) GeV when $t_{p} \rightarrow t h(b W)$ is dominant.

In this study, we use a fast detector simulator, Delphes, and therefore our result should be regarded as order estimate. Especially the discovery potential of the top partner dominantly decaying into th depends on b-tagging/mis-tagging efficiencies. They must be experimentally determined. Some of our estimate is fragile. For example, in the lower panel, when the top partner is lighter than $500 \mathrm{GeV}$, some regions are still constrained even if the decay mode $t_{p} \rightarrow t h$ is forbidden. These constraints are due to the mis-tagging of light-jets, which are regarded as b-jets, and are expected to suffer from large uncertainty of our Monte-Carlo simulations. However, these regions are covered by analyses of single- and two-b-jet channels and the ambiguities do not affect the search of the top partner.

We also consider the capability of the LHC experiment to discover the signal with the center of mass energy of $8 \mathrm{TeV}$. In the single-b-jet and multi-b-jet channels, signal events can deviate from those of the SM backgrounds at $5 \sigma$ level when their corresponding cross sections (times acceptances) satisfy following conditions,

$$
(\text { Cross section } \times \text { Acceptance })>\left\{\begin{array}{cl}
36 \mathrm{fb}: & 1 \text { lepton }+\geq 1 \text { b-jet } \\
3.6 \mathrm{fb}: & 1 \text { lepton }+\geq 2 \mathrm{~b} \text {-jets } \\
2.3 \mathrm{fb}: & 1 \text { lepton }+\geq 3 \text { b-jets. }
\end{array}\right.
$$

The region in which the top partner signal could be discovered at $5 \sigma$ level with the use of the three-b-jet channels is plotted in Fig [6 for various values of $m_{t p}$. No region for the discovery of the top partner with the mass greater than $400 \mathrm{GeV}$ can be 

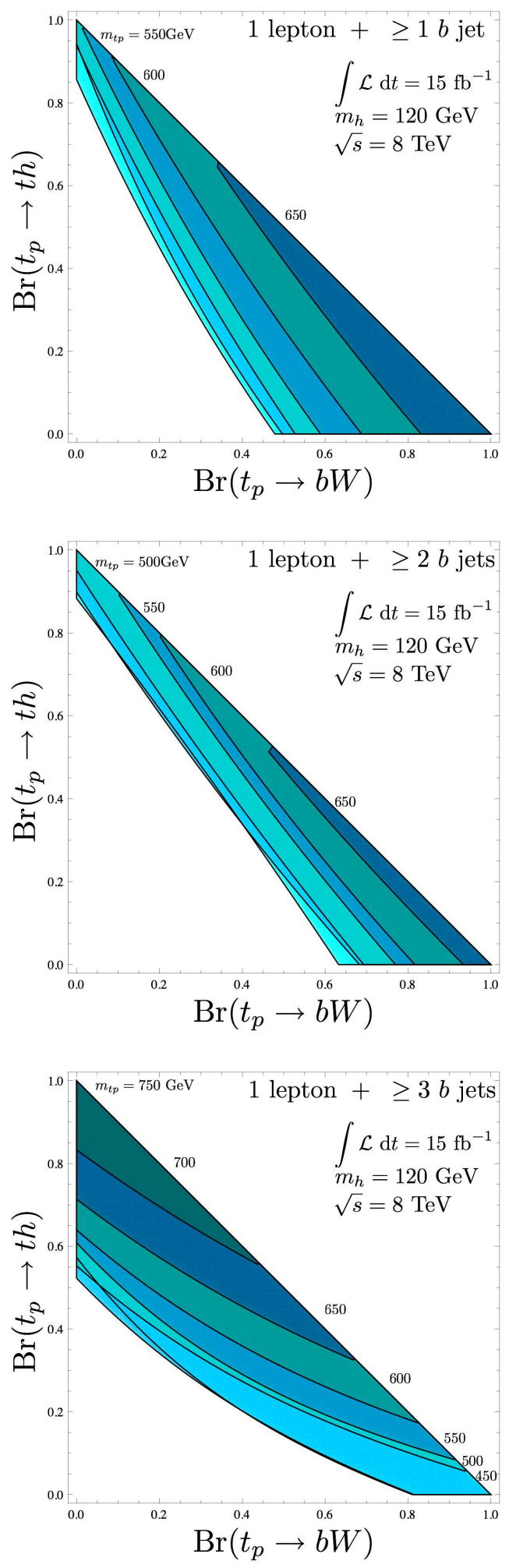

Figure 5: Regions which would be excluded by the analysis ( 1 lepton $+\geq 1$ b-jet) and that of the multi-b-jet channels ( 1 lepton $+\geq 2$ or 3 b-jets) at $95 \%$ C.L. with the integrated luminosity of $15 \mathrm{fb}^{-1}$. Contours are plotted in the region of $\operatorname{Br}\left(t_{p} \rightarrow b W\right)+\operatorname{Br}\left(t_{p} \rightarrow t h\right) \leq 1$. The center of mass energy is $8 \mathrm{TeV}$. 


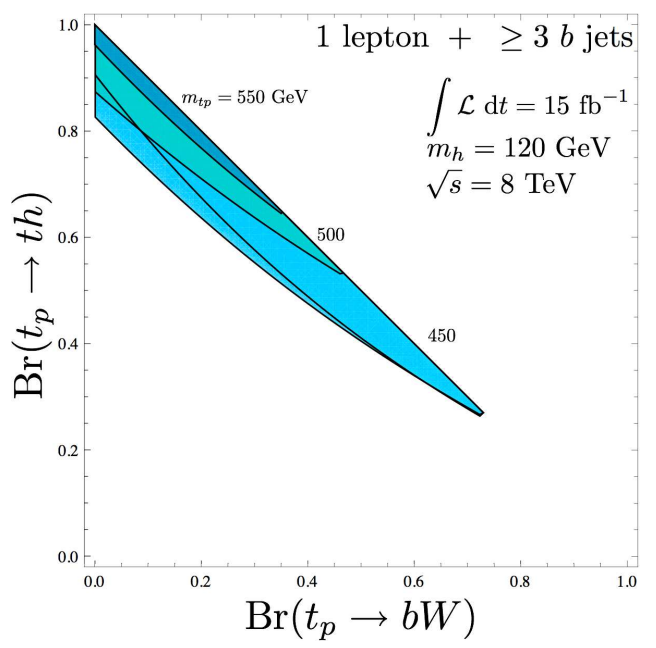

Figure 6: The region in which the signal could be discovered in the three-b-jet channels (1 lepton $+\geq 3$ b-jets) at the $5 \sigma$ level with the integrated luminosity of $15 \mathrm{fb}^{-1}$.

found in the analyses of the single- and two-b-jet channels. With the integrated luminosity of $15 \mathrm{fb}^{-1}$ at $8 \mathrm{TeV}$, the LHC experiment has the capability to the signal up to $m_{t p}<550 \mathrm{GeV}$ when the decay mode $t_{p} \rightarrow$ th dominants.

\section{Implications to new physics models}

We discuss some implications of the results obtained in the previous section to new physics models beyond the SM. Based on the effective action of the top partner given in Section 2, we clarify parameter regions which can be covered by the LHC at 8 $\mathrm{TeV}$ with $15 \mathrm{fb}^{-1}$ data.

The expected exclusion region at 95\% C.L. and the discovery region at $5 \sigma$ level are shown in Fig.7 on the $\left(m_{t p}, \sin \theta_{t L}\right)$-plane, which are obtained from analyses of all the single-, two-, and three-b-jet channels. As explainded in Section 2 and as seen in Fig.1, the rise of the right-handed mixing angle $\sin \theta_{t R}$ which strongly depends on the coefficient of the higher dimensional operator $\lambda / \Lambda$ significantly enhances the branching fraction of $t_{p} \rightarrow t h$. The $\sin \theta_{t R}$ is fixed to be $0(0.1)$ in the left (right) panel of the figure. Parameter regions which have already been constrained by the electroweak precision measurement [3] and the CMS experiment [28] are also depicted in the panels. These figures show the LHC experiment will cover the top partner mass up to about $620(750) \mathrm{GeV}$ when $\sin \theta_{t R}=0(0.1)$. In the little higgs model, 

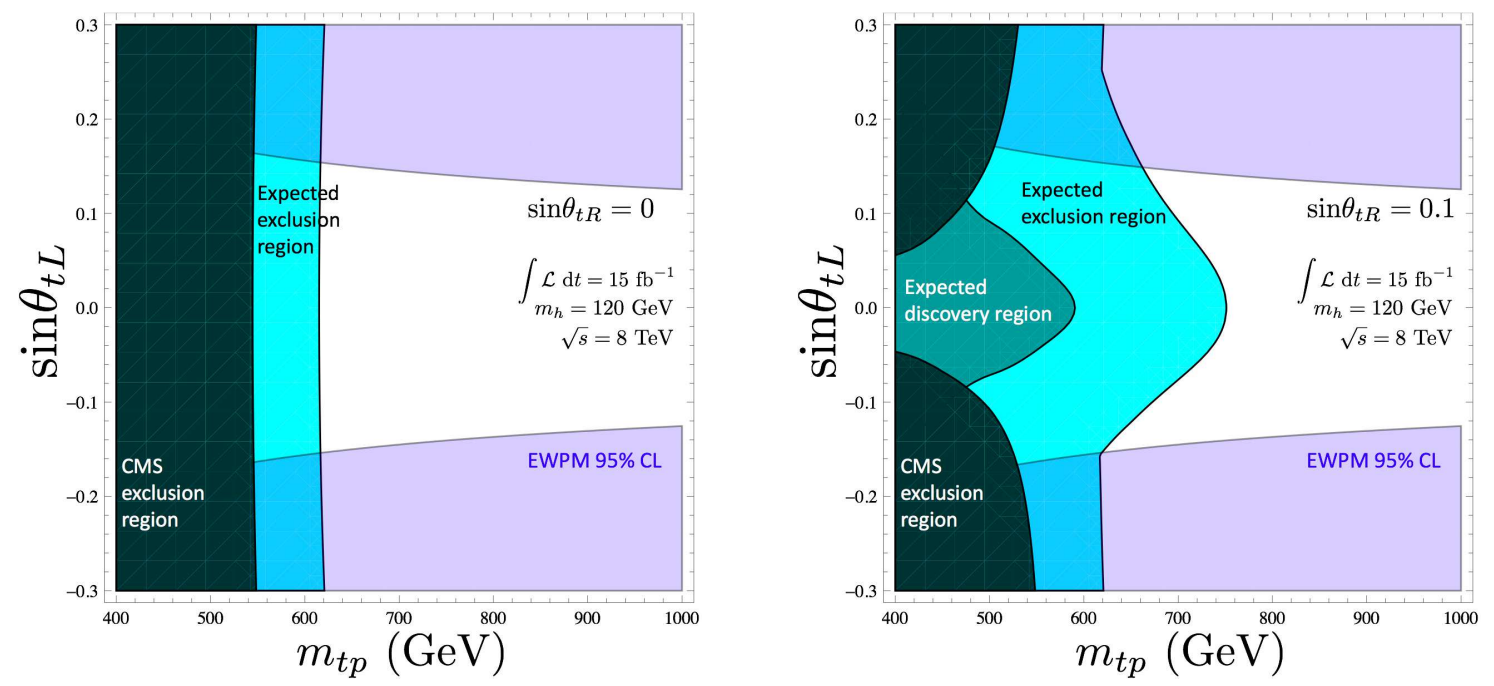

Figure 7: Expected exclusion region at 95\% C.L. and discovery region at $5 \sigma$ level. The angle $\sin \theta_{t R}$ is $0(0.1)$ in the left (right) panel.

the top partner mass larger than $600 \mathrm{GeV}$ requires the fine tuning severer than $10 \%$ [3, 29]. Also, we show the discovery region in the right panel when the mixing angle $\sin \theta_{t R}=0.1$. This indicates that the multi-b-jet channels have potential to search for the top partner at the LHC.

\section{Summary}

We have investigated the capability of the LHC experiment to search for the top partner with the center of mass energy $8 \mathrm{TeV}$ and the integrated luminosity of 15 $\mathrm{fb}^{-1}$. In this paper we assume that the top partner is the vector-like quark which is singlet (triplet) under the $\mathrm{SM} \mathrm{SU}(2)_{L}\left(\mathrm{SU}(3)_{c}\right)$ gauge group and is interacting only with the third generation quarks. In order for the discussion to be as general as possible, we have used the effective action to describe the physics of the top partner. If new physics comes from the strong dynamics, the dimension-five operator in Eq.(1) becomes sizable, which enhances the decay mode of $t_{p} \rightarrow t h$. The $t_{p} \rightarrow t h$ decay produces multiple b-jets in the final state. We have found that the multi-b-jet channels play a complementary role to the existing searches in the one-b-jet + one lepton channel and multi-jet + three leptons channel which rely on the decay modes $t_{p} \rightarrow b W$ and $t_{p} \rightarrow t Z$.

It also has been shown that the multi-b-jet channels are so powerful that it is even 
possible to discover the top partner signal at $5 \sigma$ level when the top partner lighter than about $550 \mathrm{GeV}$ dominantly decays into th. With the use of results obtained in these analyses, we have also discussed some implications to new physics models. Using all the single-, two-, and three-b-jet channels as summarized in Fig,7, we have found that wide regions of the model parameter space will be excluded at 95\% C.L. if no signal is detected. The mass of top partner is, in fact, covered up to about 750 $\mathrm{GeV}$ for the large the right-handed mixing angle $\sin \theta_{t R}$ while up to about $620 \mathrm{GeV}$ for the small $\sin \theta_{t R}$.

\section{Acknowledgement}

We thank Shoji Asai, Hitoshi Murayama, Satoshi Shirai, Satoru Yamashita and Tsutomu T. Yanagida for valuable discussions. This work is supported by Grantin-Aid for Scientific research from the Ministry of Education, Science, Sports, and Culture (MEXT), Japan (Nos. 23740169, 22244021 for S.M. and Nos. 22540300, 23104006 for M.M.N.), and also by World Premier International Research Center Initiative (WPI Initiative), MEXT, Japan. The work of K.H. and K.T. is supported in part by JSPS Research Fellowships for Young Scientists.

\section{A Validating our simulation framework}

In order to validate our simulation framework, we present the the transverse mass distributions of the SM processes. These distributions are compared to those provided by the ATLAS collaboration in Ref.[30], with particularly focusing on events involving missing transverse momentum, b-jets, and a lepton. The validation with this channel is important since we also treat the same objects in our analysis given in Sec. 3. Selection-criteria, which are imposed in order to compare with the ATLAS result, are given by

1. There is only one electron (muon) which should have $p_{T}>25(20) \mathrm{GeV}$.

2. There are at least four jets which are required to have $p_{T}>50 \mathrm{GeV}$.

3. At least one of the jets is b-tagged with $p_{T}>50 \mathrm{GeV}$ and $|\eta|>2.5$.

4. Transverse missing energy $\mathbb{E}_{T}$ should be larger than $80 \mathrm{GeV}$.

5. The transverse mass $m_{T}$ should be larger than $100 \mathrm{GeV}$. 

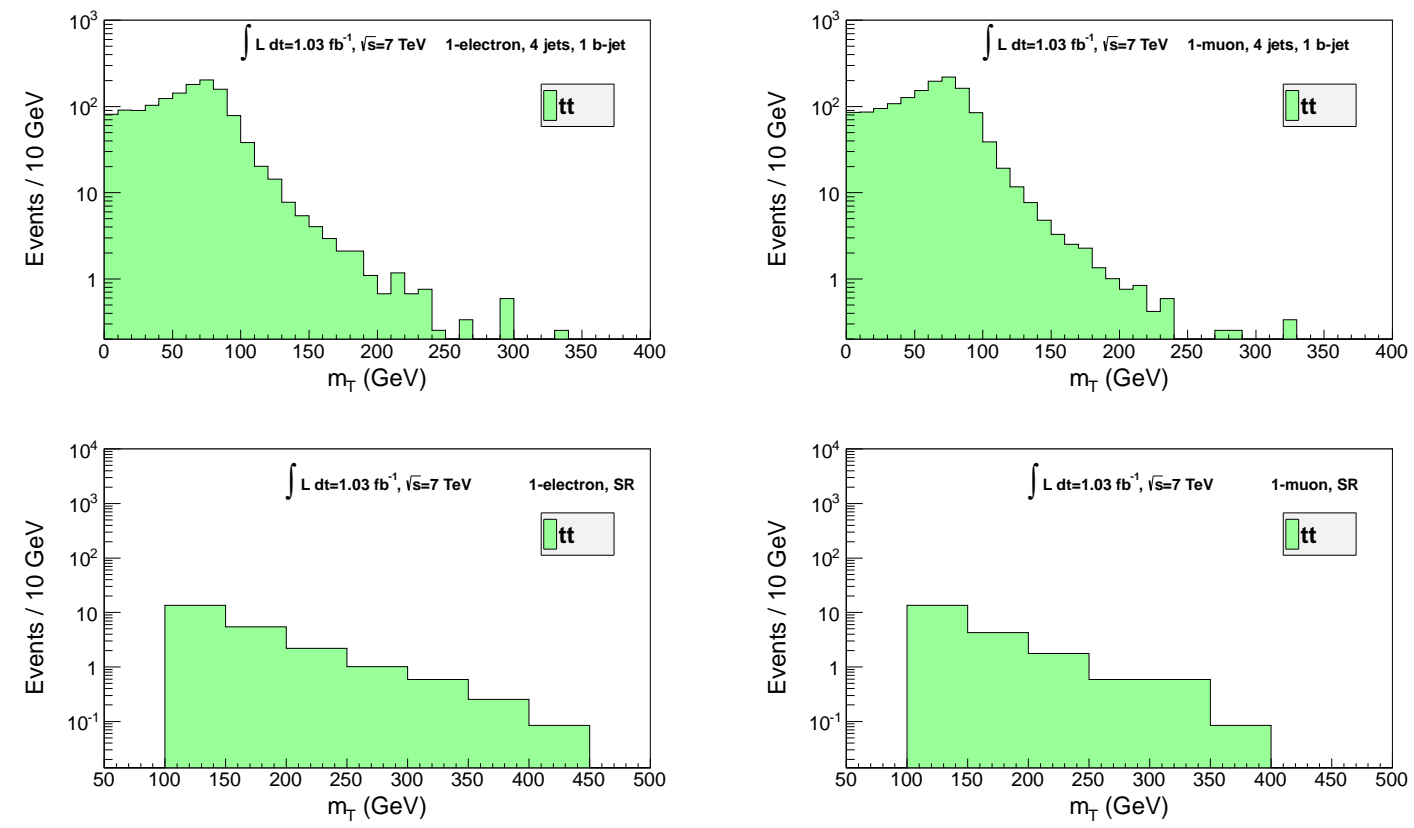

Figure 8: Distributions of the transverse mass after imposing kinematical cuts 1-3 (upper two panels) and cuts 1-6 (lower two panels). The left two panels show distributions involving an electron, while the right two panels show those involving a muon.

6. The effective mass $M_{\text {eff }}$ should be larger than $600 \mathrm{GeV}$.

The effective mass $M_{\text {eff }}$ is defined by the scalar-sum of the missing energy, the transverse momenta of leading four jets, and that of the lepton, while the transverse mass $m_{T}$ is calculated from the transverse missing momentum and the lepton momentum. In the analysis conducted by the ATLAS collaboration, jets pointing to the region where the electromagnetic calorimeter does not work properly are removed. The loss of the acceptance caused by this selection is as large as $20 \%$. We take this effect into account just by multiplying the number of events after the selections by 0.8 .

The distributions of the transverse mass after imposing kinematical cuts 1-3 and after cuts 1-6 are plotted in upper two panels and lower two panels of Fig, 8 , respectively. The distributions of $t \bar{t}+$ jets events are depicted in the figures. The left two plots show distributions involving an electron, while right two plots show those with a muon. These figures should be compared to Fig. 3 and 5 in Ref. [30]. Our results are consistent with those given by the ATLAS collaboration within the accuracy of 10-20\%. 


\section{B CMS-based analysis}

In this appendix, we show results based on the analysis in the ' 1 lepton $+\geq 1$ b-jet' channel using the kinematical variables $M_{\text {fit }}$ introduced in the CMS-analysis. We impose a following selection-criteria to generated events subsequantly,

1. There is only one lepton which is required to have $p_{T}>30 \mathrm{GeV}$.

2. Transverse missing energy $\mathbb{E}_{T}$ should be larger than $20 \mathrm{GeV}$.

3. There are more than three jets, and at least one of them are b-tagged.

4. Leading four jets are required to have $p_{T}>80,50,30$, and $30 \mathrm{GeV}$.

5. The leading b-jet is required to have $p_{T}>300 \mathrm{GeV}$.

6. The kinematical variable $M_{\text {fit }}$ should be larger than $350 \mathrm{GeV}$

7. The effective mass $M_{\text {eff }}$ should be larger than $1300 \mathrm{GeV}$.

Here, the effective mass is defined by $M_{\text {eff }}=p_{T \text {-lepton }}+\mathbb{E}_{T}+\sum_{i=1}^{\min \left(5, N_{\text {jets }}\right)} p_{T \text {-ith j }}$. The kinematical variable $M_{\text {fit }}$ is defined as $M_{\text {fit }}=\min \left(m_{l \nu b}, m_{j j b}\right)$, where the two invariant masses are calculated from all possible combination of jets which minimize following chi-2 function,

$$
\chi^{2}=\left(\frac{m_{l v}-m_{W}}{\Delta m_{l v}}\right)^{2}+\left(\frac{m_{j j}-m_{W}}{\Delta m_{j j}}\right)^{2}+\left(\frac{m_{l \nu b}-m_{j j b}}{\left[\left(\Delta m_{l \nu b}\right)^{2}+\left(\Delta m_{j j b}\right)^{2}\right]^{1 / 2}}\right)^{2},
$$

where uncertainties of the invariant mass $\Delta m_{i j}\left(\Delta m_{i j k}\right)$ are evaluated by considering the resolutions of hadronic and electromagnetic calorimeters of the ATLAS detector. The transverse momentum of the neutrino is identified with the missing transverse momentum, while the longitudinal one is treated as a free parameter which is determined by minimizing the $\chi^{2}$-function. When only one b-jet is detected, one of the other jets is regarded as another b-jet and is used to evaluate the above $\chi^{2}$-function.

The distribution of $M_{\text {fit }}$ after applying kinematical cuts 1-4 is plotted in the left panel of Fig 9 for $m_{t p}=500 \mathrm{GeV}$. Other distributions such as $p_{T-1 \mathrm{stb}}$ and $M_{\text {eff }}$ are exactly the same as those in Fig 2, The $M_{\text {fit }}$ distribution of $t \bar{t}+$ jets events is also plotted in the same figure. On the other hand, in Table 9, the cut flows of both signal and background events are shown. Numbers of events for the signal and background in the second low correspond to those assuming the integrated luminosity of $15 \mathrm{fb}^{-1}$, and their acceptances after applying all kinematical cuts are shown in the last low. Acceptances of the signal events for various masses of the top partner in each decay pattern of $t_{p} \bar{t}_{p}$ are also found in Table 10. 


\begin{tabular}{c|ccccc} 
& $t \bar{t}$ & $W+$ jets & $T \bar{T} \rightarrow b W b W$ & $T \bar{T} \rightarrow b W t h$ & $T \bar{T} \rightarrow$ thth \\
\hline Without cuts & 3060000 & 4800000 & 7650 & 7650 & 7650 \\
Cuts 1-3 & 282861 & 16756 & 1336 & 1652 & 1693 \\
Cut 4 & 133099 & 6392 & 1160 & 1555 & 1654 \\
Cut 5 & 986 & 302 & 285 & 251 & 125 \\
Cut 6 & 422 & 172 & 198 & 86 & 23 \\
Cut 7 & 102 & 65 & 60 & 28 & 8 \\
\hline Acceptance & 0.000033 & 0.000014 & 0.0079 & 0.0037 & 0.0011 \\
\hline
\end{tabular}

Table 9: Cut flows of signal and background events in the '1 lepton $+\geq 1$ b-jet' channel adopted in the CMS experiment. The mass of the top partner is $m_{t p}=500 \mathrm{GeV}$. Results of $t_{p} \bar{t}_{p} \rightarrow b W b W, b W t h$ and thth are shown separately assuming all $t_{p} \bar{t}_{p}$-decays in each mode.

Using this CMS-based analysis and assuming the systematic uncertainty of $20 \%$, the exclusion-limit on the production cross section of $t_{p} \bar{t}_{p}$ is given by

$$
(\text { Cross section } \times \text { Acceptance })>2.4 \mathrm{fb}: 1 \text { lepton }+\geq 1 \text { b-jet (CMS-based) } .
$$

The regions which would be constrained by the conventional analysis is plotted in the right panel of Fig 9 with the integrate luminosity of $15 \mathrm{fb}^{-1}$. The region is depicted on the plane of $\operatorname{Br}\left(t_{p} \rightarrow b W\right)$ and $\operatorname{Br}\left(t_{p} \rightarrow t h\right)$ for various values of the top partner mass. It can be seen that the conventional analysis gives milder constraints than those discussed in Sec. 3. On the other hand, the condition that signal events can deviate from those of SM backgrounds at $5 \sigma$ level is given by

$$
(\text { Cross section } \times \text { Acceptance })>11 \mathrm{fb}: 1 \text { lepton }+\geq 1 \mathrm{~b} \text {-jet (CMS-based). }
$$

Note that the analysis given here is not exactly the same one as the CMS one. In the CMS-analysis, high- $p_{T}$ cut on the b-jet (cut 5) is not imposed. Furthermore, the CMS collaboration puts the bound on the cross section of the signal by examining the distributions of $M_{\text {fit }}$ and $M_{\text {eff }}$, not by the number counting. However, the analysis given here is sufficient in order to compare the sensitivity of the analysis using $M_{\text {fit }}$ with the one using $M_{\mathrm{bl} \nu}$.

\section{References}

[1] N. Arkani-Hamed, A. G. Cohen, H. Georgi, Phys. Lett. B513, 232-240 (2001), and, for a review, see M. Perelstein, Prog. Part. Nucl. Phys. 58, 247 (2007). 

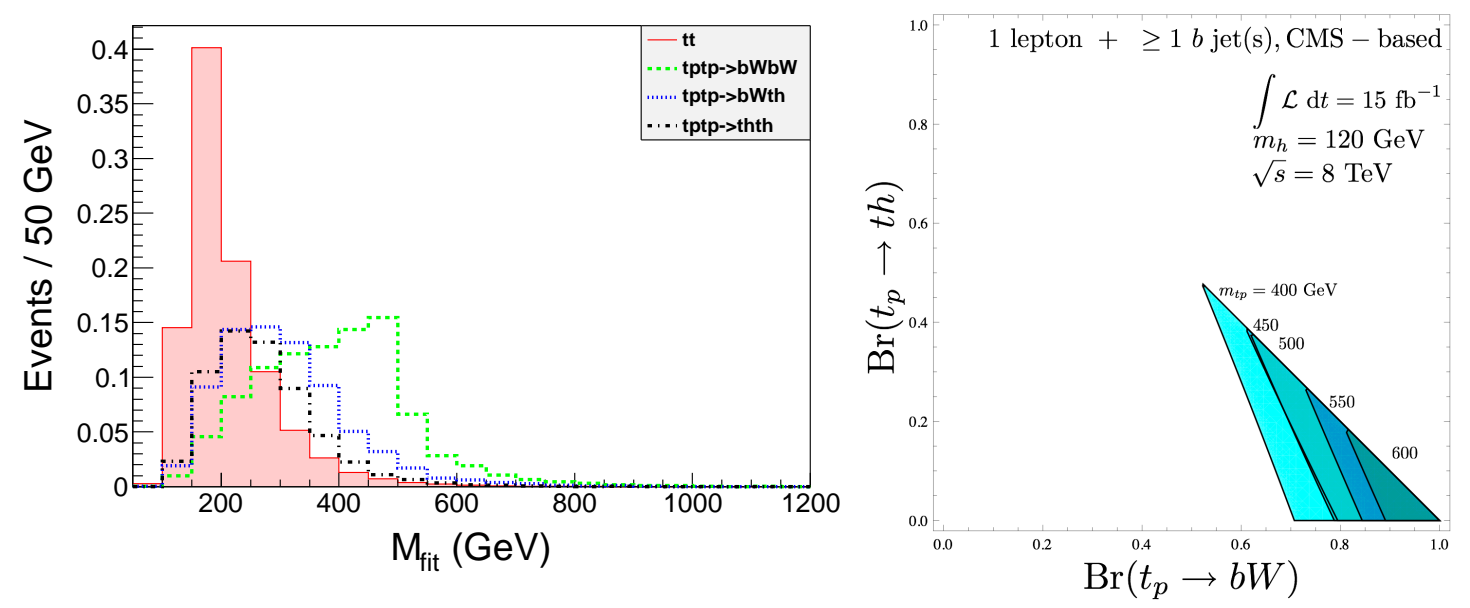

Figure 9: (Left panel) Distribution of $M_{\text {fit }}$ for signal and background $(t \bar{t}+$ jets $)$ events after applying the cuts $1-4$ in the ' 1 lepton $+\geq 1$ b-jet' channel adopted in the CMS experiment. The mass of the top partner is $m_{t p}=500 \mathrm{GeV}$. Results of $t_{p} \bar{t}_{p} \rightarrow b W b W, b W t h$ and thth are shown separately assuming all $t_{p} \bar{t}_{p}$-decays in each mode. The distribution is normalized so that its integrated value becomes unity. (Right panel) Region which would be excluded by the conventional analysis with the integrated luminosity of $15 \mathrm{fb}^{-1}$.

[2] For recent analysis, see R. Barbieri, A. Pomarol, R. Rattazzi, A. Strumia, Nucl. Phys. B703, 127-146 (2004); Z. Han, W. Skiba, Phys. Rev. D71, 075009 (2005).

[3] K. Harigaya, S. Matsumoto, M. M. Nojiri and K. Tobioka, JHEP 1201, 135 (2012).

[4] T. Moroi and Y. Okada, Mod. Phys. Lett. A 7, 187 (1992); Phys. Lett. B 295, 73 (1992); K. S. Babu, I. Gogoladze and C. Kolda, hep-ph/0410085; K. S. Babu, I. Gogoladze, M. U. Rehman and Q. Shafi, Phys. Rev. D 78, 055017 (2008); S. P. Martin, Phys. Rev. D 81, 035004 (2010); M. Asano, T. Moroi, R. Sato and T. T. Yanagida, Phys. Lett. B 705, 337 (2011).

[5] The ATLAS Collaboration, arXiv:1202.1408 [hep-ex].

[6] S. Chatrchyan et al. [The CMS Collaboration], arXiv:1202.1488 [hep-ex].

[7] Y. Okada, M. Yamaguchi and T. Yanagida, Prog. Theor. Phys. 85, 1 (1991); J. R. Ellis, G. Ridolfi and F. Zwirner, Phys. Lett. B 257, 83 (1991); H. E. Haber and R. Hempfling, Phys. Rev. Lett. 66, 1815 (1991).

[8] M. Endo, K. Hamaguchi, S. Iwamoto and N. Yokozaki, Phys. Rev. D 84, 075017 (2011); T. Moroi, R. Sato and T. T. Yanagida, arXiv:1112.3142 [hep- 


\begin{tabular}{c|cccccccc} 
Mass $(\mathrm{GeV})$ & 400 & 450 & 500 & 550 & 600 & 650 & 700 & 750 \\
\hline$T \bar{T} \rightarrow b W b W$ & 0.0025 & 0.0040 & 0.0079 & 0.013 & 0.021 & 0.029 & 0.042 & 0.051 \\
$T \bar{T} \rightarrow b W t h$ & 0.00093 & 0.0020 & 0.0037 & 0.0057 & 0.0094 & 0.014 & 0.020 & 0.030 \\
$T \bar{T} \rightarrow$ thth & 0.00048 & 0.00065 & 0.0011 & 0.0019 & 0.0031 & 0.0050 & 0.0080 & 0.011 \\
\hline
\end{tabular}

Table 10: Acceptance of the signal events in the conventional analysis adopted in the CMS experiment. Masses of the top partner are chosen between $400 \mathrm{GeV}$ and $750 \mathrm{GeV}$. Results of $t_{p} \bar{t}_{p} \rightarrow b W b W, b W t h$ and $t h t h$ are shown separately assuming all $t_{p} \bar{t}_{p}$-decays in each mode.

ph]; M. Endo, K. Hamaguchi, S. Iwamoto and N. Yokozaki, arXiv:1112.5653 [hep-ph].

[9] T. Yanir, JHEP 0206, 044 (2002); A. Arhrib and W. -S. Hou, JHEP 0607, 009 (2006); A. K. Alok, A. Dighe and D. London, Phys. Rev. D 83, 073008 (2011).

[10] The CMS Collabolation, CMS-PAS-EXO-11-099.

[11] S. Chatrchyan et al. [The CMS Collaboration], Phys. Rev. Lett. 107, 271802 (2011).

[12] J.A. Aguilar-Saavedra, JHEP 0612, 033 (2006);

[13] A. Azatov, O. Bondu, A. Falkowski, M. Felcini, S. Gascon-Shotkin, D.K. Ghosh, G. Moreau, A.Y. Rodriguez-Marrero and S. Sekmen, arXiv:1204.0455 [hep-ph].

[14] B.A. Dobrescu, K. Kong and R. Mahbubani, JHEP 0906, 001 (2009); G. Cacciapaglia, A. Deandrea, L. Panizzi, N. Gaur, D. Harada and Y. Okada, JHEP 1203, $070(2012)$

[15] N.D. Christensen and C. Duhr, Comput.Phys.Commun. 180:1614-1641 (2009).

[16] J. Alwall, M. Herquet, F. Maltoni, O. Mattelaer and T. Stelzer, JHEP 1106, 128 (2011).

[17] T. Sjostrand, S. Mrenna and P. Z. Skands, JHEP 0605, 026 (2006).

[18] S. Ovyn, X. Rouby and V. Lemaitre, arXiv:0903.2225 [hep-ph].

[19] M. Aharrouche, et al, the ATLAS Electromagnetic Barrel Calorimeter Collaboration, arXiv:0608012v1 [physics.ins-det]; Y. A. Kulchitsky, M. V. Kuzmin, J. A. Budagov, V. B. Vinogradov and M. Nessi, arXiv:0004009 [hep-ex].

[20] For example, see G. Aad et al. [The ATLAS Collaboration], Phys. Rev. D 85, 012006 (2012.) 
[21] M. Cacciari and G. P. Salam, Phys. Lett. B 641, 57 (2006); M. Cacciari, G. P. Salam and G. Soyez, JHEP 0804, 063 (2008).

[22] The ATLAS Collaboration, ATLAS-CONF-2011-089.

[23] The ATLAS Collaboration, CERN-LHCC-99-14.

[24] The ATLAS Collaboration, arXiv:0901.0512.

[25] M. Aliev, H. Lacker, U. Langenfeld, S. Moch, P. Uwer and M. Wiedermann, Comput. Phys. Commun. 182, 1034 (2011).

[26] The CMS Collabolation, CMS-PAS-EXO-11-051.

[27] G. Choudalakis, arXiv:1110.5295 [hep-ph].

[28] The CMS Collabolation, CMS-PAS-EXO-11-050.

[29] C. Csaki, J. Hubisz, G.D. Kribs, P. Meade, J. Terning, Phys. Rev. D 67, 115002 (2003).

[30] The ATLAS Collaboration, ATLAS-CONF-2011-130. 